\title{
A Remark on the Fractional Integral Operators and the Image Formulas of Generalized Lommel-Wright Function
}

\author{
Ritu Agarwal ${ }^{1 *}$, Sonal Jain ${ }^{2}$, Ravi P. Agarwal ${ }^{3}$ and Dumitru Baleanu ${ }^{4,5}$ \\ ${ }^{1}$ Department of Mathematics, Malaviya National Institute of Technology, Jaipur, India, ${ }^{2}$ Department of Mathematics, Faculty \\ of Applied Science, Madhav Institue of Technology \& Sciences, Gwalior, India, ${ }^{3}$ Department of Mathematics, Texas A\&M \\ University, Kingsville, TX, United States, ${ }^{4}$ Department of Mathematics, Çankaya University, Ankara, Turkey, ${ }^{5}$ Institute of \\ Space Science, Bucharest, Romania
}

In this paper, the operators of fractional integration introduced by Marichev-SaigoMaeda involving Appell's function $F_{3}(\cdot)$ are applied, and several new image formulas of generalized Lommel-Wright function are established. Also, by implementing some integral transforms on the resulting formulas, few more image formulas have been presented. We can conclude that all derived results in our work generalize numerous well-known results and are capable of yielding a number of applications in the theory of special functions.

\section{OPEN ACCESS}

Edited by:

Carla M. A. Pinto,

Instituto Superior de Engenharia do

Porto (ISEP), Portugal

Reviewed by:

Bruce J. West,

United States Army Research

Laboratory, United States

Carlo Cattani,

Università degli Studi della Tuscia, Italy

${ }^{*}$ Correspondence:

Ritu Agarwal

ragarwal.maths@mnit.ac.in

Specialty section

This article was submitted to

Mathematical Physics,

a section of the journal

Frontiers in Physics

Received: 23 May 2018

Accepted: 05 July 2018

Published: 14 August 2018

Citation:

Agarwal R, Jain S, Agarwal RP and

Baleanu D (2018) A Remark on the

Fractional Integral Operators and the

Image Formulas of Generalized

Lommel-Wright Function.

Front. Phys. 6:79

doi: 10.3389/fphy.2018.00079
Primary: 44A20 Transforms of special functions; 65R10 For numerical methods; 26A33 Fractional derivatives and integrals; Secondary: 33C20 Generalized hypergeometric series, pFq; 33E50 Special functions in characteristic p (gamma functions, etc.); 2010 AMS classification by MathSciNet

Keywords: Marichev-Saigo-Maeda fractional integral operators, generalized Lommel-Wright function, generalized Fox-Wright function, generalized hypergeometric series, integral transform

\section{INTRODUCTION AND PRELIMINARIES}

Fractional calculus (FC) represents a complex physical phenomenon in a more accurate and efficient way than classical calculus. In recent years, many researchers [1-7] have used fractional order integral models in real-world problems in various fields of science and technology. There exists several definitions of fractional order integrals in the literature that can be used to solve the fractional integral equations involving special functions. For an exhaustive literature review, one may refer to the paper by Srivastava and Saxena [8].

The generalized functions such as Bessel, Lommel, Struve, and Lommel-Wright functions have originated from concrete problems in applied fields of sciences viz mechanics, physics, engineering, astronomy, etc.

The generalized Lommel-Wright function $J_{\omega, \vartheta}^{\varphi, m}(z)$ is defined by de'Oteiza et al. [9] and is represented in the following manner:

$$
\begin{aligned}
& J_{\omega, \vartheta}^{\varphi, m}(z)=(z / 2)^{\omega+2 \vartheta} \sum_{k=0}^{\infty} \frac{(-1)^{k}(z / 2)^{2 k}}{(\Gamma(\vartheta+k+1))^{m} \Gamma(\omega+k \varphi+\vartheta+1)} \\
&=(z / 2)^{\omega+2 \vartheta}{ }_{1} \psi_{m+1}[(1,1) ; \underbrace{(\vartheta+1,1)}_{m-\text { times }}, \quad(\omega+\vartheta+1, \varphi) ;-z^{2} / 4] \\
& z \in \mathbb{C} \backslash(-\infty, 0], \quad \varphi>0, \quad m \in \mathbb{N}, \quad \omega, \vartheta \in \mathbb{C},
\end{aligned}
$$


where ${ }_{p} \psi_{q}$ denotes the Fox-Wright generalized hypergeometric function which is defined as given in Srivastava and Karlsson [10, p. 21] and Kilbas et al. [11, P. 56]

$$
{ }_{p} \psi_{q}\left(\begin{array}{l}
\left(a_{1}, A_{1}\right), \ldots\left(a_{p}, A_{p}\right) ; \\
\left(b_{1}, B_{1}\right), \ldots,\left(b_{q}, B_{q}\right) ;
\end{array}\right)=\sum_{n=0}^{\infty} \frac{\Pi_{j=1}^{p} \Gamma\left(a_{j}+n A_{j}\right)}{\Pi_{j=1}^{q} \Gamma\left(b_{j}+n B_{j}\right)} \frac{z^{n}}{n !},
$$

where $a_{i}, b_{j} \in \mathbb{C}$ and $A_{i}, B_{j} \in \mathbb{R}=(-\infty, \infty) ; A_{i}, B_{j} \neq 0$, $i=1,2, \ldots, p, j=1,2, \ldots, q, \sum_{j=1}^{q} B_{j}-\sum_{j=1}^{p} A_{j}>-1$.

A useful generalization of the Lommel-Wright function and its special cases, $J_{\omega}^{\varphi}(z) J_{\omega, \vartheta}^{\varphi}(z)$, depending on the arbitrary fractional parameter $\varphi>0$ presents a fractional order extension of the Bessel function $J_{\omega}(z)$.

Prieto et al. [12] studied some useful results in the theory of fractional calculus operators of generalized Lommel-Wright function. The convergence of series involving generalized Lommel-Wright function was studied by Konovska [13].

When $m=1$, the following generalization of the Bessel function, introduced by Pathak [14] is obtained as a special case of generalized Lommel-Wright function (1.1) (see e.g., [15, p. 353]):

$$
\begin{aligned}
J_{\omega, \vartheta}^{\varphi}(z)= & J_{\omega, \vartheta}^{\varphi, 1}(z) \\
= & \left(\frac{z}{2}\right)^{\omega+2 \vartheta} \sum_{k=0}^{\infty} \frac{(-1)^{k}\left(\frac{z}{2}\right)^{2 k}}{\Gamma(\vartheta+k+1) \Gamma(\omega+k \varphi+\vartheta+1)},(1, \\
& z \in \mathbb{C} \backslash(-\infty, 0], \varphi>0, \quad \omega, \vartheta \in \mathbb{C} .
\end{aligned}
$$

On taking $m=1, \varphi=1$, and $\vartheta=\frac{1}{2}$ in (1.1), we obtain the Struve function $H_{\omega}(\cdot)$ (see e.g., [16, p. 28, Equation (1.170)])

$$
\begin{aligned}
H_{\omega}(z) & =J_{\omega, 1 / 2}^{1,1} \\
& =\left(\frac{z}{2}\right)^{\omega+1} \sum_{k=0}^{\infty} \frac{(-1)^{k}\left(\frac{z}{2}\right)^{2 k}}{\Gamma\left(k+\frac{3}{2}\right) \Gamma\left(k+\omega+\frac{3}{2}\right)} z, \omega \in \mathbb{C} .
\end{aligned}
$$

If we take $m=1, \varphi=1$, and $\vartheta=0$ in (1.1), it gives the relationship with the Bessel function as follows (see e.g., [16, p. 27, Equation (1.161)]):

$$
\begin{aligned}
J_{\omega}(z)=J_{\omega, 0}^{1,1}(z)=\sum_{k=0}^{\infty} \frac{(-1)^{k}(z / 2)^{\omega+2 k}}{\Gamma(\omega+k+1) k !} \\
\quad z, \omega \in \mathbb{C}, \quad z \neq 0, \quad \Re(\omega)>-1 .
\end{aligned}
$$

A generalization of the hypergeometric fractional integrals, including the Saigo operators $[17,18]$ has been introduced by Marichev [19]. The details of these fractional operators have been found in Samko et al. [5, p. 194, Equation (10.47)] and later extended and studied by Saigo and Maeda [20, p. 393, Equation (4.12) and Equation (4.13)] in terms of complex order Appell function $F_{3}(\cdot)$ of two variables (see $[10$, p. 23]) in the kernel

$$
\begin{aligned}
& F_{3}\left(\zeta, \zeta^{\prime}, \varrho, \varrho^{\prime} ; \eta ; x ; y\right) \\
& \quad=\sum_{m, n=0}^{\infty} \frac{(\zeta)_{m}\left(\zeta^{\prime}\right)_{n}(\varrho)_{m}\left(\varrho^{\prime}\right)_{n}}{(\eta)_{m+n}} \frac{x^{m}}{m !} \frac{y^{n}}{n !}, \quad(\max \{|x|,|y|\}<1) .
\end{aligned}
$$

The Appell function $F_{3}$ reduces to the Gauss hypergeomatric function ${ }_{2} F_{1}$ and satisfies the system of two linear partial differential equations of the second order as follows (see [10, p. 301, Equation 9.4]):

$$
F_{3}(\zeta, \eta-\zeta, \varrho, \eta-\varrho ; \eta ; x ; y)={ }_{2} F_{1}(\zeta, \varrho ; \eta ; x+y-x y) .
$$

Further, it is easy to see that

$$
F_{3}\left(\zeta, 0, \varrho, \varrho^{\prime}, \eta ; x, y\right)={ }_{2} F_{1}(\zeta, \varrho ; \eta ; x)
$$

and

$$
F_{3}\left(0, \zeta^{\prime}, \varrho, \varrho^{\prime}, \eta ; x, y\right)={ }_{2} F_{1}\left(\zeta^{\prime}, \varrho^{\prime} ; \eta ; y\right)
$$

In this paper, we develop and study the image formulas involving the generalized Lommel-Wright function using fractional calculus integral operators. We use the generalized MarichevSaigo-Maeda fractional integral operators, involving the Appell function, defined as follows:

$$
\begin{aligned}
\left(I_{0+}^{\zeta, \zeta^{\prime}, \varrho, \varrho^{\prime}, \kappa} f\right)(x)= & \frac{x^{-\zeta}}{\Gamma \kappa} \int_{0}^{x}(x-t)^{\kappa-1} t^{-\zeta^{\prime}} \\
& \times F_{3}\left(\zeta, \zeta^{\prime}, \varrho, \varrho^{\prime} ; \kappa ; 1-\frac{t}{x}, 1-\frac{x}{t}\right) f(t) d t \\
& \Re(\kappa)>0, \zeta, \zeta^{\prime}, \varrho, \varrho^{\prime}, \kappa \in \mathbb{C}, x>0
\end{aligned}
$$

and

$$
\begin{aligned}
& \left(I_{0-}^{\zeta, \zeta^{\prime}, \varrho, \varrho^{\prime}, \kappa} f\right)(x) \\
& =\frac{x^{-\zeta}}{\Gamma \kappa} \int_{x}^{\infty}(t-x)^{\kappa-1} t^{-\zeta} F_{3}\left(\zeta, \zeta^{\prime}, \varrho, \varrho^{\prime} ; \kappa ; 1-\frac{x}{t}, 1-\frac{t}{x}\right) f(t) d t, \\
& \quad \Re(\kappa)>0, \zeta, \zeta^{\prime}, \varrho, \varrho^{\prime}, \kappa \in \mathbb{C}, x>0 .
\end{aligned}
$$

respectively.

The power functions of left-hand sided and right-hand sided Marichev-Saigo-Maeda fractional integral operators as given in the Equations (1.10) and (1.11) (see Saigo et al. [6, 20]) are given by

$$
\begin{aligned}
& \left(I_{0+}^{\zeta, \zeta^{\prime}, \varrho, \varrho^{\prime}, \kappa} t^{\chi-1}\right)(x) \\
& =\frac{\Gamma(\chi) \Gamma\left(\chi+\kappa-\zeta-\zeta^{\prime}-\varrho\right) \Gamma\left(\chi+\varrho^{\prime}-\zeta^{\prime}\right)}{\Gamma\left(\chi+\varrho^{\prime}\right) \Gamma\left(\chi+\kappa-\zeta-\zeta^{\prime}\right) \Gamma\left(\chi+\kappa-\zeta^{\prime}-\varrho\right)} x^{\chi+\kappa-\zeta-\zeta^{\prime}-1}
\end{aligned}
$$

where $\zeta, \zeta^{\prime}, \varrho, \varrho^{\prime}, \kappa \in \mathbb{C}, x>0$ and if $\Re(\kappa)>0, \Re(\chi)>$ $\max \left\{0, \Re\left(\zeta+\zeta^{\prime}+\varrho-\kappa\right), \Re\left(\zeta^{\prime}-\varrho^{\prime}\right)\right\}$.

$$
\begin{aligned}
& \left(I_{0-}^{\zeta, \zeta^{\prime}, \varrho, \varrho^{\prime}, \kappa} t^{\chi-1}\right)(x) \\
& =\frac{\Gamma\left(1-\chi-\kappa+\zeta+\zeta^{\prime}\right) \Gamma\left(1-\chi+\zeta+\varrho^{\prime}-\kappa\right) \Gamma(1-\chi-\varrho)}{\Gamma(1-\chi) \Gamma\left(1-\chi+\zeta+\zeta^{\prime}+\varrho+\varrho^{\prime}-\kappa\right) \Gamma(1-\chi+\zeta-\varrho)} \\
& \quad \times x^{\chi-\zeta-\zeta^{\prime}+\kappa-1},
\end{aligned}
$$

where $\zeta, \zeta^{\prime}, \varrho, \varrho^{\prime}, \kappa \in \mathbb{C}$ are such that $\Re(\kappa)>0$ and $\Re(\chi)<$ $1+\min \left\{\Re(-\varrho), \Re\left(\zeta+\zeta^{\prime}-\kappa\right), \Re\left(\zeta+\varrho^{\prime}-\kappa\right)\right\}$. 


\subsection{Relation Among the Operators}

In this section, we recall some relationships between the fractional integral operators.

If we set $\zeta^{\prime}=0$ then in view of the formula (1.8), the relationship between Marichev-Saigo-Maeda and the Saigo fractional integral operators is found by Saxena and Saigo [6, p. 93, Equation (2.15)] as

$$
\left(I_{0, x}^{\zeta, 0, \varrho, \varrho^{\prime}, \eta} f\right)(x)=\left(I_{0, x}^{\eta, \zeta-\eta,-\varrho} f\right)(x), \quad(\Re(\eta)>0)
$$

and

$$
\left(I_{x, \infty}^{\zeta, 0, \varrho, \varrho^{\prime}, \eta} f\right)(x)=\left(I_{x, \infty}^{\eta, \zeta-\eta,-\varrho} f\right)(x), \quad(\Re(\eta)>0)
$$

where the general operators $I_{0, x}^{\zeta, 0, \varrho, \varrho^{\prime}, \eta}$ and $I_{0, x}^{\zeta, 0, \varrho, \varrho^{\prime}, \eta}$ reduce, respectively, to the Saigo operators $I_{0, x}^{\zeta, \varrho, \eta}$ and $I_{x, \infty}^{\zeta, \varrho, \eta}$ [17] defined as follows:

$$
\begin{aligned}
\left(I_{0, x}^{\zeta, \varrho, \eta} f\right)(x)= & \frac{x^{-\zeta-\varrho}}{\Gamma(\zeta)} \int_{0}^{x}(x-t)_{2}^{\zeta-1} \\
& \times F_{1}\left(\zeta+\varrho,-\eta ; \zeta ; 1-\frac{t}{x}\right) f(t) d t, \quad(\Re(\zeta)>0)
\end{aligned}
$$

and

$$
\begin{aligned}
\left(I_{x, \infty}^{\zeta, \varrho, \eta} f\right)(x)= & \int_{x}^{\infty}(t-x)^{\zeta-1} t^{-\zeta-\varrho_{2}} \\
& \times F_{1}\left(\zeta+\varrho,-\eta ; \zeta ; 1-\frac{x}{t}\right) f(t) d t, \quad(\Re(\zeta)>0)
\end{aligned}
$$

where integrals in (1.16) and (1.17) exist.

Let $\zeta, \varrho, \eta, \chi \in \mathbb{C}$ with $\Re(\zeta)>0$. Then the following power function formulas involving the Saigo operators hold true:

$$
\begin{gathered}
\left(I_{0, x}^{\zeta, \varrho, \eta} t^{\chi-1}\right)(x)=\frac{\Gamma(\chi) \Gamma(\chi+\eta-\varrho)}{\Gamma(\chi-\varrho) \Gamma(\chi+\eta+\zeta)} x^{\chi-\varrho-1}, \\
\Re(\chi)>\max \{0, \Re(\varrho-\eta)\}
\end{gathered}
$$

and

$$
\begin{gathered}
\left(I_{x, \infty}^{\zeta, \varrho, \eta} t^{\chi-1}\right)(x)=\frac{\Gamma(1-\chi+\varrho) \Gamma(1-\chi+\eta)}{\Gamma(1-\chi) \Gamma(1-\chi+\zeta+\varrho+\eta)} x^{\chi-\varrho-1} \\
(\Re(\chi)<1+\min \{\Re(\varrho), \Re(\eta)\}) .
\end{gathered}
$$

On replacing $\varrho=-\zeta$ in the operators $I_{0, x}^{\zeta, \varrho, \eta}(\cdot)$ and $I_{x, \infty}^{\zeta, \varrho, \eta}(\cdot)$, these reduce to the Riemann-Liouville and the Weyl fractional integral operators, respectively, by means of the following relationships (see Kilbas [11]):

$$
\left(\mathcal{R}_{0, x}^{\zeta} f\right)(x)=\left(I_{0, x}^{\zeta,-\zeta, \eta} f\right)(x)
$$

and

$$
\left(\mathcal{W}_{x, \infty}^{\zeta} f\right)(x)=\left(I_{x, \infty}^{\zeta,-\zeta, \eta} f\right)(x)
$$

The Riemann-Liouville fractional integral operator and the Weyl fractional integral operator are defined as follows (see e.g., [21]):

$$
\left(\mathcal{R}_{0, x}^{\zeta} f\right)(x)=\frac{1}{\Gamma(\zeta)} \int_{0}^{x}(x-t)^{\zeta-1} f(t) d t, \quad(\Re(\zeta)>0)
$$

and

$$
\left(\mathcal{W}_{x, \infty}^{\zeta} f\right)(x)=\frac{1}{\Gamma(\zeta)} \int_{0}^{x}(t-x)^{\zeta-1} f(t) d t, \quad(\Re(\zeta)>0)
$$

provided both the integrals converge.

The operators $I_{0, x}^{\zeta, Q, \eta}(\cdot)$ and $I_{x, \infty, \infty}^{\zeta, Q, \eta}(\cdot)$ reduce to Erdélyi-Kober fractional integral operators on setting $\varrho=0$ as follows:

$$
\left(\mathcal{E}_{0, x}^{\zeta, \eta} f\right)(x)=\left(I_{0, x}^{\zeta, 0, \eta} f\right)(x)
$$

and

$$
\left(\mathcal{K}_{x, \infty}^{\zeta, \eta} f\right)(x)=\left(I_{0, x}^{\zeta, 0, \eta} f\right)(x)
$$

where the Erdélyi-Kober type fractional integral operators are defined as follows (see [22]):

$$
\left(\mathcal{E}_{0, x}^{\zeta, \eta} f\right)(x)=\frac{x^{-\zeta-\eta}}{\Gamma(\zeta)} \int_{0}^{x}(x-t)^{\zeta-1} t^{\eta} f(t) d t, \quad(\Re(\zeta)>0)
$$

and

$$
\left(\mathcal{K}_{x, \infty}^{\zeta, \eta} f\right)(x)=\frac{x^{\eta}}{\Gamma(\zeta)} \int_{x}^{\infty}(t-x)^{\zeta-1} t^{-\zeta-\eta} f(t) d t, \quad(\Re(\zeta)>0)
$$

The function $f(t)$ is constrained so that both the defining integrals (1.26) and (1.27) converge.

The Beta transform (see, e.g. [23]) of a complex valued function $f(t)$ of a real variable $t$ is defined as follows:

$$
\begin{aligned}
& B\{f(t): a, b\}=\int_{0}^{1} t^{a-1}(1-t)^{b-1} f(t) d t, \\
& \Re(t)>0, \mathfrak{R}(a), \mathfrak{R}(b)>0 .
\end{aligned}
$$

Beta transform of the power function $t^{\chi-1}$ is given by:

$$
\begin{aligned}
& B\left\{t^{\chi-1} ; a, b\right\}=\int_{0}^{1} t^{a+\chi-2}(1-t)^{b-1} d t \\
&=\frac{\Gamma(a+\chi-1) \Gamma(b)}{\Gamma(a+\chi+b-1)}, \Re(t)>0, \Re(a), \Re(b)>0 . \\
&(1.29)
\end{aligned}
$$

The $P_{\delta}$ - transform of a complex valued function $f(t)$ of a real variable $t$ denoted by $P_{\delta}[f(t) ; s]$ is a function $F(s)$ of a complex 
variable $s$, valid under certain conditions on $f(t)$, (given below is defined as (see Kumar [24])

$$
P_{\delta}[f(t) ; s]=F(s)=\int_{0}^{\infty}[1+(\delta-1) s]^{-\frac{t}{\delta-1}} f(t) d t, \quad \delta>1 .
$$

Here $f(t)$ as a function is integrable over any finite interval $(a, b)$, $0<a<t<b$; there exists a real number $c$ such that

(i) if $b>0$ is arbitrary, then $\int_{b}^{\Upsilon} e^{-c t} f(t) d z$ tends to a finite limit as $\Upsilon \rightarrow \infty$

(ii) for arbitrary $a>0, \int_{\omega}^{a}|f(t) d t|$ tends to a finite limit as $\omega \rightarrow 0+$, then the $P_{\delta}$-transform $P_{\delta}[f(t) ; s]$ exists for $\Re\left(\frac{\ln [1+(\delta-1) s]}{\delta-1}\right)>c$ for $s \in \mathbb{C}$.

$P_{\delta}$ - transform of the power function $t^{\chi-1}$ is given by

$$
\begin{aligned}
P_{\delta}\left[z^{\chi-1} ; s\right]= & \left\{\frac{\delta-1}{\ln [1+(\delta-1) s]}\right\}^{\chi} \Gamma(\chi), \\
& \chi \in \mathbb{C}, \Re(\chi)>0, \quad \delta>1 .
\end{aligned}
$$

$P_{\delta}$-transform has found many applications. The pathway transforms are the paths going from the binomial form $\ln [1+(\delta-1) s]^{-\frac{t}{\delta-1}}$ to the exponential from $e^{-s t}$. In $P_{\delta^{-}}$ transform, the variable $\mathrm{t}$ is shifted from the binomial factor $\ln [1+(\delta-1) s]^{-\frac{t}{\delta-1}}$ to the exponent, Hence, this form is more suitable for obtaining translation, convolution, etc. Recently, Agarwal et al. [25] found the solution of non-homogeneous time fractional heat equation and fractional Volterra integral equation using integral transform of pathway type. Also, Srivastava et al. [26] and [27] found some results involving generalized hypergeometric function and generalized incomplete gamma function by using $P_{\delta}$-transform.

If we take $\delta \rightarrow 1$ in Equation (1.30), the $P_{\delta}$-transform reduces to Laplace integral transform (Sneddon [23]):

$$
L[f(t) ; s]=\int_{0}^{\infty} e^{-t s} f(t) d z ;, \quad \Re(s)>0 .
$$

The following relationship between the $P_{\delta}$-transform is defined by (1.30) and the classical Laplace transform is defined by (1.32)
The following integral formula involving the Whittaker function (see Mathai et al. [16, p. 56]) is used in finding the image formula:

$$
\begin{gathered}
\int_{0}^{\infty} t^{\tau-1} e^{-\frac{t}{2}} W_{\sigma, \eta}(t) d t=\frac{\Gamma\left(\tau+\eta+\frac{1}{2}\right) \Gamma\left(\tau-\eta+\frac{1}{2}\right)}{\Gamma\left(\tau-\sigma+\frac{1}{2}\right)}, \\
(\sigma \in \mathbb{C}, \quad \Re(\tau \pm \eta)>-1 / 2) .
\end{gathered}
$$

The Whittaker function (see e.g., Mathai et al. [16, p. 22]) is defined by

$$
\begin{aligned}
W_{\sigma, \eta}(z)= & \frac{\Gamma(-2 \eta)}{\Gamma\left(\frac{1}{2}-\sigma-\eta\right)} M_{\sigma, \eta}(z)+\frac{\Gamma(2 \eta)}{\Gamma\left(\frac{1}{2}-\sigma+\eta\right)} M_{\sigma,-\eta}(z) \\
= & W_{\sigma,-\eta}(z) \\
& \quad \sigma \in \mathbb{C}, \quad \Re(1 / 2+\eta \pm \sigma)>0
\end{aligned}
$$

where

$$
\begin{array}{r}
M_{\sigma, \eta}(z)=z^{\eta+\frac{1}{2}} e^{-\frac{z}{2}}{ }_{1} F_{1}\left(\frac{1}{2}-\sigma+\eta ; 2 \eta+1 ; z\right), \\
\Re(1 / 2+\eta \pm \sigma)>0, \quad|\arg z|<\pi .
\end{array}
$$

\section{IMAGE FORMULA ASSOCIATED WITH FRACTIONAL INTEGRAL OPERATORS}

Here, we establish image formulas for the generalized LommelWright function involving Saigo-Maeda fractional integral operators (1.10) and (1.11), in terms of the Fox-Wright function.

Theorem 2.1. Let $\zeta, \zeta^{\prime}, \varrho, \varrho^{\prime}, \kappa, \vartheta \in \mathbb{C}, m \in \mathbb{N}, \varphi>0$ and $x>0$ be such that

$$
\begin{array}{r}
\mathfrak{N}(\kappa)>0, \quad \mathfrak{R}(\omega)>-1, \\
\Re(\chi+\omega)>\max \left\{0, \mathfrak{R}\left(\zeta+\zeta^{\prime}+\varrho-\kappa\right), \mathfrak{R}\left(\zeta^{\prime}-\varrho^{\prime}\right)\right\}
\end{array}
$$

then there holds the formula

$$
\begin{aligned}
& I_{0+}^{\zeta, \zeta^{\prime}, \varrho, \varrho^{\prime}, \kappa}\left[t^{\chi-1} J_{\omega, \vartheta}^{\varphi, m}(t z)\right](x)=x^{A-\zeta-\zeta^{\prime}+\kappa-1}\left(\frac{z}{2}\right)^{\omega+2 \vartheta} \\
& { }_{4} \psi_{4+m}\left[\begin{array}{c}
(A, 2),\left(A+\kappa-\zeta-\zeta^{\prime}-\varrho, 2\right),\left(A+\varrho^{\prime}-\zeta^{\prime}, 2\right),(1,1) \\
\left(A+\varrho^{\prime}, 2\right),\left(A+\kappa-\zeta-\zeta^{\prime}, 2\right),\left(A+\kappa-\zeta^{\prime}-\varrho, 2\right),(\omega+\vartheta+1, \varphi), \underbrace{(\vartheta+1,1)}_{m-\text { times }}
\end{array} \mid-\frac{(z x)^{2}}{4}\right]
\end{aligned}
$$

$$
P_{\delta}[f(t): s]=L\left[f(t): \frac{\ln [1+(\delta-1) s]}{\delta-1}\right], \quad(\delta>1)
$$

or, equivalently,

$$
L[f(t): s]=P_{\delta}\left[f(t): \frac{e^{(\delta-1) s}-1}{\delta-1}\right], \quad(\delta>1),
$$

which can be applied to convert the table of Laplace transforms into the corresponding table of $P_{\delta}$-transforms and vice versa. where $A=\chi+\omega+2 \vartheta$.

Proof: Under the conditions stated with the Theorem 2.1, by taking the fractional integral of (1.1) using the equation (1.10) therein and changing the order of integration and summation, we get

$$
\begin{aligned}
I_{0+}^{\zeta, \zeta^{\prime}, \varrho, \varrho^{\prime}, \kappa}\left[t^{\chi-1} J_{\omega, \vartheta}^{\varphi, m}(t z)\right](x) \\
=\sum_{k=0}^{\infty} \frac{(-1)^{k}\left(\frac{z}{2}\right)^{\omega+2 \vartheta+2 k} \Gamma(k+1)}{(\Gamma(\vartheta+k+1))^{m} \Gamma(\omega+k \varphi+\vartheta+1) k !} \\
\quad \times I_{0+}^{\zeta, \zeta^{\prime}, \varrho, \varrho^{\prime}, \kappa}\left(t^{\omega+2 \vartheta+2 k+\chi-1}\right)(x)
\end{aligned}
$$


Further, applying the result (1.12) with $\chi$ replaced by $\chi+\omega+$ $2 \vartheta+2 k$, we obtain

$$
\begin{aligned}
& I_{0+}^{\zeta, \zeta^{\prime}, \varrho, \varrho^{\prime}, \kappa}\left[t^{\chi-1} J_{\omega, \vartheta}^{\varphi, m}(t z)\right](x) \\
& =x^{A-\zeta-\zeta^{\prime}+\kappa-1}\left(\frac{z}{2}\right)^{\omega+2 \vartheta} \sum_{k=0}^{\infty} \frac{(-1)^{k} \Gamma(A+2 k) \Gamma(k+1)}{\Gamma\left(A+\varrho^{\prime}+2 k\right)(\Gamma(\vartheta+1+k))^{m}} \\
& \quad \times \frac{\Gamma\left(A+\kappa-\zeta-\zeta^{\prime}-\varrho+2 k\right) \Gamma\left(A+\varrho^{\prime}-\zeta^{\prime}+2 k\right)}{\Gamma\left(A+\kappa-\zeta^{\prime}-\varrho+2 k\right) \Gamma(\omega+\vartheta+1+\varphi k) \Gamma\left(A+\kappa-\zeta-\zeta^{\prime}+2 k\right)} \\
& \quad \times \frac{(z x)^{2 k}}{4^{k} k !}
\end{aligned}
$$

Here $A=\chi+\omega+2 \vartheta$.

Interpreting the right-hand side of the above equation, in view of the definition (1.2), we arrive at the result (2.2).

Theorem 2.2. Let $\zeta, \zeta^{\prime}, \varrho, \varrho^{\prime}, \kappa, \vartheta \in \mathbb{C}, m \in \mathbb{N}, \varphi>0$ and $x>0$ be such that

$$
\begin{gathered}
\Re(\kappa)>0, \quad \Re(\omega)>-1, \\
\Re(\chi-\omega)>1+\min \left\{\Re(-\varrho), \Re\left(\zeta+\zeta^{\prime}-\kappa\right), \Re\left(\zeta+\varrho^{\prime}-\kappa\right)\right\}
\end{gathered}
$$

then there holds the formula

$$
\left.\begin{array}{l}
I_{0-}^{\zeta, \zeta^{\prime}, \varrho, \varrho^{\prime}, \kappa}\left[t^{\chi-1} J_{\omega, \vartheta}^{\varphi, m}(z / t)\right](x)=x^{\kappa-\zeta-\zeta^{\prime}-A}\left(\frac{z}{2}\right)^{\omega+2 \vartheta} \\
{ }_{4} \psi_{4+m}\left[\begin{array}{c}
\left(A-\kappa+\zeta+\zeta^{\prime}, 2\right),\left(A+\zeta+\varrho^{\prime}-\kappa, 2\right), \\
(A-\varrho, 2),(1,1) \\
(A, 2)\left(A+\zeta+\zeta^{\prime}+\varrho^{\prime}-\kappa, 2\right),(A+\zeta-\varrho, 2), \\
(\omega+\vartheta+1, \varphi), \underbrace{(\vartheta+1,1)}_{m-\text { times }}
\end{array} \mid-\frac{z^{2}}{4 x^{2}}\right.
\end{array}\right]
$$

where $A=1-\chi+\omega+2 \vartheta$.

Proof: Under the conditions stated with the Theorem 2.2, on making use of the definitions (1.11) and (1.1) and changing the order of integration and summation, we have

$$
\begin{aligned}
I_{0-}^{\zeta, \zeta^{\prime}, \varrho, \varrho^{\prime}, \kappa}\left[t^{\chi-1} J_{\omega, \vartheta}^{\varphi, m}(z / t)\right](x) & \sum_{k=0}^{\infty} \frac{(-1)^{k}\left(\frac{z}{2}\right)^{\omega+2 \vartheta+2 k} \Gamma(k+1)}{(\Gamma(\vartheta+k+1))^{m} \Gamma(\omega+k \varphi+\vartheta+1) k !} \\
& \times I_{0-}^{\zeta, \zeta^{\prime}, \varrho, \varrho^{\prime}, \kappa}\left(t^{\chi-\omega-2 \vartheta-2 k-1}\right)(x)
\end{aligned}
$$

Here, on applying the formula (1.13) with $\chi$ replaced by $\chi-\omega-$ $2 \vartheta-2 k$, we obtain

$$
\begin{aligned}
& I_{0-}^{\zeta, \zeta^{\prime}, \varrho, \varrho^{\prime}, \kappa}\left[t^{\chi-1} J_{\omega, \vartheta}^{\varphi, m}(z / t)\right](x) \\
& =x^{\kappa-\zeta-\zeta^{\prime}-A}\left(\frac{z}{2}\right)^{\omega+2 \vartheta} \sum_{k=0}^{\infty} \frac{(-1)^{k} \Gamma(A-\varrho+2 k)}{\Gamma(A+2 k)(\Gamma(\vartheta+k+1))^{m}} \\
& \quad \times \frac{\Gamma(k+1) \Gamma\left(A-\kappa+\zeta+\zeta^{\prime}+2 k\right) \Gamma\left(A+\zeta+\varrho^{\prime}-\kappa+2 k\right)}{\Gamma(A+\zeta-\varrho+2 k) \Gamma(\omega+k \varphi+\vartheta+1) \Gamma\left(A+\zeta+\zeta^{\prime}+\varrho^{\prime}-\kappa+2 k\right)} \\
& \quad \times \frac{(z)^{2 k}}{\left(4 x^{2}\right)^{k} k !}
\end{aligned}
$$

where $A=1-\chi+\omega+2 \vartheta$.
So in view of the definition of the generalized Lommel-Wright function given by (1.1), the Equation (2.8) leads to the result (2.6).

For $m=1$ and in the light of Equation (1.3), Theorem 2.1 leads to the following corollaries:

Corollary 2.1. Under the conditions stated with the Equation (2.1), the following image formula

$$
\begin{aligned}
& I_{0+}^{\zeta, \zeta^{\prime}, \varrho, \varrho^{\prime}, \kappa}\left[t^{\chi-1} J_{\omega, \vartheta}^{\varphi, 1}(z t)\right](x)=x^{A-\zeta-\zeta^{\prime}+\kappa-1}\left(\frac{z}{2}\right)^{\omega+2 \vartheta} \\
& \times{ }_{4} \psi_{5}\left[\begin{array}{c}
(A, 2),\left(A+\kappa-\zeta-\zeta^{\prime}-\varrho, 2\right), \\
\left(A+\varrho^{\prime}-\zeta^{\prime}, 2\right),(1,1) \\
\left(A+\varrho^{\prime}, 2\right),\left(\begin{array}{c}
\left(A+\kappa-\zeta-\zeta^{\prime}, 2\right),\left(A+\kappa-\zeta^{\prime}-\varrho, 2\right), \\
(\omega+\vartheta+1, \varphi),(\vartheta+1,1)
\end{array}\right.
\end{array} \mid-\frac{(z x)^{2}}{4}\right]
\end{aligned}
$$

$A=\chi+\omega+2 \vartheta$, for generalized Bessel function $J_{\omega, \vartheta}^{\varphi, 1}(z t)$ holds true.

Corollary 2.2. Under the conditions stated with the Equation (2.5), the image formula

$$
\begin{aligned}
& I_{0-}^{\zeta, \zeta^{\prime}, \varrho, \varrho^{\prime}, \kappa}\left[t^{\chi-1} J_{\omega, \vartheta}^{\varphi, 1}(z / t)\right](x) \\
& =x^{\kappa-\zeta-\zeta^{\prime}-A}\left(\frac{z}{2}\right)^{\omega+2 \vartheta} \\
& \times{ }_{4} \psi_{5}\left[\begin{array}{c|c}
\left(A-\kappa+\zeta+\zeta^{\prime}, 2\right),\left(A+\zeta+\varrho^{\prime}-\kappa, 2\right), & \\
(A-\varrho, 2),(1,1) & \\
(A, 2)\left(A+\zeta+\zeta^{\prime}+\varrho^{\prime}-\kappa, 2\right),(A+\zeta-\varrho, 2), & -\frac{z^{2}}{4 x^{2}} \\
(\omega+\vartheta+1, \varphi),(\vartheta+1,1) &
\end{array}\right]
\end{aligned}
$$

$A=1-\chi+\omega+2 \vartheta$, for generalized Bessel function $J_{\omega, \vartheta}^{\varphi, 1}(z / t)$ holds true.

If we take $m=1, \varphi=1$, and $\vartheta=\frac{1}{2}$ in (2.2), then we obtain the corresponding results for the Struve function $H_{\omega}(\cdot)$ [16] as

Corollary 2.3. Under the conditions stated with the Equation (2.1), the following image formula

$$
\begin{aligned}
& I_{0+}^{\zeta, \zeta^{\prime}, \varrho, \varrho^{\prime}, \kappa}\left[t^{\chi-1} H_{\omega}(z t)\right](x) \\
& =x^{A-\zeta-\zeta^{\prime}+\kappa-1}\left(\frac{z}{2}\right)^{\omega+1} \\
& \times{ }_{4} \psi_{5}\left[\begin{array}{c}
(A, 2),\left(A+\kappa-\zeta-\zeta^{\prime}-\varrho, 2\right), \\
\left(A+\varrho^{\prime}-\zeta^{\prime}, 2\right),(1,1) \\
\left(A+\varrho^{\prime}, 2\right),\left(A+\kappa-\zeta-\zeta^{\prime}, 2\right), \\
\left(A+\kappa-\zeta^{\prime}-\varrho, 2\right),\left(\omega+\frac{3}{2}, 1\right),\left(\frac{3}{2}, 1\right)
\end{array} \mid-\frac{(z x)^{2}}{4}\right]
\end{aligned}
$$

$A=\chi+\omega+1$, for Struve function $H_{\omega}(z t)$ holds true. 
Corollary 2.4. Under the conditions stated with the Equation (2.5), the following image formula

$$
\begin{aligned}
& I_{0-}^{\zeta, \zeta^{\prime}, \varrho, \varrho^{\prime}, \kappa}\left[t^{\chi-1} H_{\omega}(z / t)\right](x) \\
& =x^{\chi-\omega-\zeta-\zeta^{\prime}+\kappa-2}\left(\frac{z}{2}\right)^{\omega+1} \\
& \times{ }_{4} \psi_{5}\left[\begin{array}{c|c}
\left(A-\kappa+\zeta+\zeta^{\prime}, 2\right),\left(A+\zeta+\varrho^{\prime}-\kappa, 2\right), & \\
(A-\varrho, 2),(1,1) & \\
(A, 2)\left(A+\zeta+\zeta^{\prime}+\varrho^{\prime}-\kappa, 2\right),(A+\zeta-\varrho, 2), & -\frac{z^{2}}{4 x^{2}} \\
\left(\omega+\frac{3}{2}, 1\right),\left(\frac{3}{2}, 1\right)
\end{array}\right]
\end{aligned}
$$

where $A=2-\chi+\omega$, for Struve function $H_{\omega}(z / t)$ holds true.

\subsection{Special Cases}

(1) On taking $\varphi=1, m=1, \vartheta=0$, and $z=1$ in Theorem 2.1, we obtain the image formula for the Bessel function considered by Purohit et al. [28, Theorem 1].

Corollary 2.5. Under the conditions stated with the Equation (2.1), the following image formula

$$
\begin{aligned}
& I_{0+}^{\zeta, \zeta^{\prime}, \varrho, \varrho^{\prime}, \kappa}\left[t^{\chi-1} J_{\omega}(t)\right](x) \\
& =\frac{x^{\chi+\omega-\zeta-\zeta^{\prime}+\kappa-1}}{2^{\omega}} \\
& \times{ }_{3} \psi_{4}\left[\begin{array}{c}
(\chi+\omega, 2),\left(\chi+\omega+\kappa-\zeta-\zeta^{\prime}-\varrho, 2\right), \\
\left(\chi+\omega+\varrho^{\prime}-\zeta^{\prime}, 2\right) \\
\left(\chi+\omega+\varrho^{\prime}, 2\right),\left(\chi+\omega+\kappa-\zeta-\zeta^{\prime}, 2\right), \\
\left(\chi+\omega+\kappa-\zeta^{\prime}-\varrho, 2\right),(\omega+1,1)
\end{array}\right.
\end{aligned}
$$

for Bessel function $J_{\omega}(t)$ holds true.

(2) Further, on taking $\varphi=1, m=1$, and $\vartheta=0$ in Theorem 2.2, we arrive the right-sided image formula for the Bessel function considered by Purohit et al. [28, Theorem 2].

Corollary 2.6. Under the conditions stated with the Equation (2.5), the image formula

$$
\begin{aligned}
I_{0-}^{\zeta, \zeta^{\prime}, \varrho, \varrho^{\prime}, \kappa}\left[t^{\chi-1} J_{\omega}(1 / t)\right](x) \\
\quad=\frac{x^{\kappa-\zeta-\zeta^{\prime}-1+\chi-\omega}}{2^{\omega}} \\
\quad \times{ }_{3} \psi_{4}\left[\begin{array}{c}
\left(1-\chi+\omega-\kappa+\zeta+\zeta^{\prime}, 2\right),\left(1-\chi+\omega+\zeta+\varrho^{\prime}-\kappa, 2\right),(1-\chi+\omega-\varrho, 2) \\
(1-\chi+\omega, 2)\left(1-\chi+\omega+\zeta+\zeta^{\prime}+\varrho^{\prime}-\kappa, 2\right),(1-\chi+\omega+\zeta-\varrho, 2),(\omega+1,1)
\end{array} \mid-\frac{1}{4 x^{2}}\right]
\end{aligned}
$$

transforms such as Beta transform, pathway transform, Laplace transform, and Whittaker transform.

\subsection{Image Formulas for Beta Transform}

Theorem 3.1. Let $\zeta, \zeta^{\prime}, \varrho, \varrho^{\prime}, \kappa, \vartheta \in \mathbb{C}, m \in \mathbb{N}, \varphi>0$, and $x>0$ be such that

$$
\begin{array}{r}
\Re(l)>0, \quad \mathfrak{R}(n)>0 \quad \mathfrak{K}(\kappa)>0, \quad \mathfrak{R}(\omega)>-1, \\
\Re(\chi+\omega)>\max \left\{0, \mathfrak{R}\left(\zeta+\zeta^{\prime}+\varrho-\kappa\right), \mathfrak{R}\left(\zeta^{\prime}-\varrho^{\prime}\right)\right\}
\end{array}
$$

then the following Beta transform formula holds:

$$
\begin{aligned}
& B\left[I_{0+}^{\zeta, \zeta^{\prime}, \varrho, \varrho^{\prime}, \kappa}\left(t^{\chi-1} J_{\omega, \vartheta}^{\varphi, m}(t z)\right)(x): l, n\right] \\
& =\frac{x^{A-\zeta-\zeta^{\prime}+\kappa-1} \Gamma(n)}{2^{\omega+2 \vartheta}} \\
& \quad{ }_{5} \psi_{5+m}\left[\begin{array}{c}
(A, 2),\left(A+\kappa-\zeta-\zeta^{\prime}-\varrho, 2\right), \\
\left(A+\varrho^{\prime}-\zeta^{\prime}, 2\right),(C-n, 2)(1,1) \\
\left(A+\varrho^{\prime}, 2\right),\left(A+\kappa-\zeta-\zeta^{\prime}, 2\right),\left(A+\kappa-\zeta^{\prime}-\varrho, 2\right), \\
(\omega+\vartheta+1, \varphi),(C, 2), \underbrace{(\vartheta+1,1)}_{m-\text { times }}
\end{array} \mid\right.
\end{aligned}
$$

Here $A=\chi+\omega+2 \vartheta$ and $C=l+\omega+2 \vartheta+n$.

Proof: For our convenience, let the left-hand side of the formula (3.2) be denoted by $\varsigma$. Applying (1.28) to Equation (3.2), we get

$$
\varsigma=\int_{0}^{1} z^{l-1}(1-z)^{n-1}\left[I_{0+}^{\zeta, \zeta^{\prime}, \varrho, \varrho^{\prime}, \kappa}\left(t^{\chi-1} J_{\omega, \vartheta}^{\varphi, m}(t z)\right)(x)\right] d z
$$

Here, applying Equation (2.2) to the integral, we obtain the following expression

$$
\begin{aligned}
\varsigma= & \int_{0}^{1} z^{l-1}(1-z)^{n-1} z^{\omega+2 \vartheta} \frac{x^{A-\zeta-\zeta^{\prime}+\kappa-1}}{2^{\omega+2 \vartheta}} \\
& \times \sum_{k=0}^{\infty} \frac{(-1)^{k} \Gamma(A+2 k) \Gamma(k+1)}{\Gamma\left(A+\varrho^{\prime}+2 k\right) \Gamma\left(A+\kappa-\zeta-\zeta^{\prime}+2 k\right)} \\
& \times \frac{\Gamma\left(A+\varrho^{\prime}-\zeta^{\prime}+2 k\right) \Gamma\left(A+\kappa-\zeta-\zeta^{\prime}-\varrho+2 k\right)}{\Gamma\left(A+\kappa-\zeta^{\prime}-\varrho+2 k\right) \Gamma(\omega+\vartheta+1+\varphi k)(\Gamma(\vartheta+1+k))^{m}} \\
& \times \frac{\left(z x^{2}\right)^{k}}{4^{k} k !} d z
\end{aligned}
$$

for Bessel function $J_{\omega}(1 / t)$ holds true.

\section{IMAGE FORMULAS ASSOCIATED WITH INTEGRAL TRANSFORMS}

In this section, we obtain the theorem involving the results obtained in previous sections associated with the integral
Here $A=\chi+\omega+2 \vartheta$.

Interchanging the order of integration and summation, we have 


$$
\begin{aligned}
\varsigma= & \frac{x^{A-\zeta-\zeta^{\prime}+\kappa-1}}{2^{\omega+2 \vartheta}} \sum_{k=0}^{\infty} \frac{\Gamma(A+2 k) \Gamma\left(A+\kappa-\zeta-\zeta^{\prime}-\varrho+2 k\right)}{\Gamma\left(A+\kappa-\zeta-\zeta^{\prime}+2 k\right) \Gamma\left(A+\kappa-\zeta^{\prime}-\varrho+2 k\right)} \\
& \times \frac{\Gamma\left(A+\varrho^{\prime}-\zeta^{\prime}+2 k\right) \Gamma(k+1)(-1)^{k}}{\Gamma\left(A+\varrho^{\prime}+2 k\right) \Gamma(\omega+\vartheta+1+\varphi k)(\Gamma(\vartheta+1+k))^{m}} \frac{\left(x^{2}\right)^{k}}{4^{k} k !} \times \int_{0}^{1} z^{l+\omega+2 \vartheta+2 k-1}(1-z)^{n-1} d z \\
= & \frac{x^{A-\zeta-\zeta^{\prime}+\kappa-1}}{2^{\omega+2 \vartheta}} \sum_{k=0}^{\infty} \frac{\Gamma(l+\omega+2 \vartheta+2 k) \Gamma(n) \Gamma(A+2 k) \Gamma\left(A+\kappa-\zeta-\zeta^{\prime}-\varrho+2 k\right)}{\Gamma(l+\omega+2 \vartheta+2 k+n) \Gamma\left(A+\varrho^{\prime}+2 k\right) \Gamma\left(A+\kappa-\zeta-\zeta^{\prime}+2 k\right)} \\
& \times \frac{\Gamma\left(A+\varrho^{\prime}-\zeta^{\prime}+2 k\right) \Gamma(k+1)}{\Gamma\left(A+\kappa-\zeta^{\prime}-\varrho+2 k\right) \Gamma(\omega+\vartheta+1+\varphi k)(\Gamma(\vartheta+1+k))^{m}} \times \frac{\left(-x^{2}\right)^{k}}{4^{k} k !}
\end{aligned}
$$

Interpreting the right-hand side of the above equation, in the view of the definition (1.2), we arrive at the required result (3.2).

Theorem 3.2. Let $\zeta, \zeta^{\prime}, \varrho, \varrho^{\prime}, \kappa, \vartheta, \omega \in \mathbb{C}, m \in \mathbb{N}, \varphi>0$, and $x>0$ be such that

$$
\begin{array}{r}
\Re(\kappa)>0, \quad \Re(\omega)>-1, \quad \Re(l)>0, \quad \Re(n)>0, \\
\Re(\chi-\omega)>1+\min \left\{\Re(-\varrho), \Re\left(\zeta+\zeta^{\prime}-\kappa\right), \Re\left(\zeta+\varrho^{\prime}-\kappa\right)\right\}
\end{array}
$$

then the following Beta transform formula holds:

$$
\begin{aligned}
& B\left[I_{0-}^{\zeta, \zeta^{\prime}, \varrho, \varrho^{\prime}, \kappa}\left(t^{\chi-1} J_{\omega, \vartheta}^{\varphi, m}(z / t)\right)(x): l, n\right\} \\
& =\frac{x^{\kappa-\zeta-\zeta^{\prime}-A} \Gamma(n)}{2^{\omega+2 \vartheta}} \\
& \times 5 \psi_{5+m}\left[\begin{array}{c}
\left(A-\kappa+\zeta+\zeta^{\prime}, 2\right),\left(A+\zeta+\varrho^{\prime}-\kappa, 2\right), \\
(A-\varrho, 2),(C-n, 2),(1,1)
\end{array} \mid\right. \\
& \begin{array}{c}
(A, 2)\left(A+\zeta+\zeta^{\prime}+\varrho^{\prime}-\kappa, 2\right),(A+\zeta-\varrho, 2), \\
(\omega+\vartheta+1, \varphi),(C, 2), \underbrace{(\vartheta+1,1)}_{m-\text { times }}
\end{array}
\end{aligned}
$$

where $A=1-\chi+\omega+2 \vartheta$ and $C=l+\omega+2 \vartheta+n$.

Proof: The proof of the fractional integral formula (3.5) is similar to the proof of the formula (3.2) given in Theorem 3.1.

\section{Remark 3.1.}

(1) For $m=1$, Theorem 3.1 and Theorem 3.2 leads to the corresponding results for fractional integral of generalized Bessel function defined by (1.3).

(2) If we take $m=1, \varphi=1$, and $\vartheta=\frac{1}{2}$ in (3.2) and (3.5), we get the corresponding results for fractional integral of Struve function defined in (1.4).

(3) On taking $m=1, \varphi=1$, and $\vartheta=0$, in (3.2) and (3.5), we get the results for fractional integral of Bessel function defined in (1.5).

\subsection{Image Formulas for $\boldsymbol{P}_{\boldsymbol{\delta}}$-Transform}

Theorem 3.3. Let $\zeta, \zeta^{\prime}, \varrho, \varrho^{\prime}, \kappa, \chi, \vartheta \in \mathbb{C}, m \in \mathbb{N}, \varphi>$ $0, \mathfrak{R}(\chi)>0, \mathfrak{R}(s)>0, \delta>1$, and $x>0$ be such that

$$
\begin{array}{r}
\Re(\kappa)>0, \quad \Re(\omega)>-1, \quad \Re(s)>0, \\
\Re(\chi+\omega)>\max \left\{0, \Re\left(\zeta+\zeta^{\prime}+\varrho-\kappa\right), \Re\left(\zeta^{\prime}-\varrho\right)\right\}
\end{array}
$$

then the following $P_{\delta}$-transform formula holds:

$$
\begin{aligned}
& P_{\delta}\left[z^{l-1}\left(I_{0+}^{\zeta, \zeta^{\prime}, \varrho, \varrho^{\prime}, \kappa} t^{\chi-1} J_{\omega, \vartheta}^{\varphi, m}(t z)\right)(x): s\right] \\
& =(\Lambda(\delta ; s))^{l+\omega+2 \vartheta} \frac{x^{A-\zeta-\zeta^{\prime}+\kappa-1}}{2^{\omega+2 \vartheta}} \\
& \times{ }_{5} \psi_{4+m}\left[\begin{array}{c}
(A, 2),\left(A+\kappa-\zeta-\zeta^{\prime}-\varrho, 2\right), \\
\left(A+\varrho^{\prime}-\zeta^{\prime}, 2\right),(l+\omega+2 \vartheta, 2),(1,1) \\
\left(A+\varrho^{\prime}, 2\right),\left(A+\kappa-\zeta-\zeta^{\prime}, 2\right), \\
\left(A+\kappa-\zeta^{\prime}-\varrho, 2\right), \\
(\omega+\vartheta+1, \varphi), \underbrace{(\vartheta+1,1)}_{m-\text { times }}
\end{array} \mid-\frac{(\Lambda(\delta ; s) x)^{2}}{4}\right.
\end{aligned} \mid
$$

where $A=\chi+\omega+2 \vartheta$ and $\Lambda(\delta ; s)=\left(\frac{\delta-1}{\ln [1+(\delta-1) s]}\right)$.

Proof: For our convenience, we let the left-hand side of the formula (3.7) be denoted as $\boldsymbol{\Xi}$. Applying (1.30) to Equation (3.2) we get,

$$
\Xi=\int_{0}^{\infty}[1+(\delta-1) s]^{-\frac{z}{\delta-1}} z^{l-1} I_{0+}^{\zeta, \zeta^{\prime}, \varrho, \varrho^{\prime}, \kappa}\left(t^{\chi-1} J_{\omega, \vartheta}^{\varphi, m}(t z)\right)(x) d z
$$

Here, applying Equation (2.4) to the integral, we obtain the following expression:

$$
\begin{aligned}
& \Xi=\frac{x^{A-\zeta-\zeta^{\prime}+\kappa-1}}{2^{\omega+2 \vartheta}} \sum_{k=0}^{\infty} \frac{(-1)^{k} \Gamma(A+2 k) \Gamma\left(A+\kappa-\zeta-\zeta^{\prime}-\varrho+2 k\right)}{\Gamma\left(A+\varrho^{\prime}+2 k\right) \Gamma\left(A+\kappa-\zeta-\zeta^{\prime}+2 k\right) \Gamma\left(A+\kappa-\zeta^{\prime}-\varrho+2 k\right)} \\
& \frac{\Gamma\left(A+\varrho^{\prime}-\zeta^{\prime}+2 k\right) \Gamma(k+1)}{\Gamma(\omega+\vartheta+1+\varphi k)(\Gamma(\vartheta+1+k))^{m}} \frac{(x)^{2 k}}{4^{k} k !} \times \int_{0}^{\infty}[1+(\delta-1) s]^{-\frac{z}{\delta-1}} z^{\omega+2 \vartheta+2 k+l-1} d z
\end{aligned}
$$


Here making use of the result (1.31) and interchanging the order of integration and summation, we obtain,
Corollary 3.2. Under the conditions stated with the Equation (3.9), the following Laplace transform formula holds true:

$$
\begin{aligned}
\Xi & =(\Lambda(\delta ; s))^{l+\omega+2 \vartheta} \frac{x^{A-\zeta-\zeta^{\prime}+\kappa-1}}{2^{\omega+2 \vartheta}} \sum_{k=0}^{\infty} \frac{\Gamma(A+2 k) \Gamma\left(A+\kappa-\zeta-\zeta^{\prime}-\varrho+2 k\right)}{\Gamma\left(A+\varrho^{\prime}+2 k\right) \Gamma\left(A+\kappa-\zeta-\zeta^{\prime}+2 k\right)} \\
& \times \frac{\Gamma(\omega+2 \vartheta+2 k+l) \Gamma\left(A+\varrho^{\prime}-\zeta^{\prime}+2 k\right) \Gamma(k+1)(-1)^{k}}{\Gamma\left(A+\kappa-\zeta^{\prime}-\varrho+2 k\right) \Gamma(\omega+\vartheta+1+\varphi k)(\Gamma(\vartheta+1+k))^{m}} \frac{\{\Lambda(\delta ; s) x\}^{2 k}}{4^{k} k !}
\end{aligned}
$$

where $A=\chi+\omega+2 \vartheta$ and $\Lambda(\delta ; s)=\left(\frac{\delta-1}{\ln [1+(\delta-1) s]}\right)$.

In view of the definition (1.2), we arrive at the required result (3.7).

Theorem 3.4. Let $\zeta, \zeta^{\prime}, \varrho, \varrho^{\prime}, \kappa, \vartheta \in \mathbb{C}, m \in \mathbb{N}, \varphi>0 \Re(\chi)>$ $0, \mathfrak{R}(s)>0, \delta>1$, and $x>0$ be such that

$$
\begin{gathered}
\Re(\kappa)>0, \Re(\omega)>-1, \Re(s)>0, \\
\Re(\chi-\omega)>1+\min \left\{\Re(-\varrho), \Re\left(\zeta+\zeta^{\prime}-\kappa\right), \Re\left(\zeta+\varrho^{\prime}-\kappa\right)\right\}
\end{gathered}
$$

then the following $P_{\delta}$-transform formula holds:

$$
\begin{aligned}
& P_{\delta}\left(z^{l-1}\left[I_{0-}^{\zeta, \zeta^{\prime}, \varrho, \varrho^{\prime}, \kappa} t^{\chi-1} J_{\omega, \vartheta}^{\varphi, m}(z / t)\right](x): s\right) \\
& =(\Lambda(\delta ; s))^{l+\omega+2 \vartheta} \frac{x^{\chi-\omega-2 \vartheta-\zeta-\zeta^{\prime}+\kappa-1}}{2^{\omega+2 \vartheta}} \\
& \times{ }_{5} \psi_{4+m}\left[\begin{array}{c}
\left(A-\kappa+\zeta+\zeta^{\prime}, 2\right),\left(A+\zeta+\varrho^{\prime}-\kappa, 2\right), \\
(A-\varrho, 2),(l+\omega+2 \vartheta, 2),(1,1) \\
(A, 2)\left(A+\zeta+\zeta^{\prime}+\varrho^{\prime}-\kappa, 2\right),(A+\zeta-\varrho, 2), \\
(\omega+\vartheta+1, \varphi), \underbrace{(\vartheta+1,1)}_{m-\text { times }}
\end{array} \mid-\frac{\{\Lambda(\delta ; s)\}^{2}}{4 x^{2}}\right]
\end{aligned}
$$

where $A=1-\chi+\omega+2 \vartheta$ and $\Lambda(\delta ; s)=\left\{\frac{\delta-1}{\ln [1+(\delta-1) s]}\right\}$.

Proof: Our demonstration of the $P_{\delta}$-transform of generalized Lommel-Wright function (3.10) is based upon the known result (2.6).

A limit case of the Theorems 3.3 and 3.4 when $\delta \rightarrow 1$ yields the following corollaries for the Laplace transform in view of the (1.32).

Corollary 3.1. Under the conditions stated with the Equation (3.6), the following Laplace transform formula holds true:

$$
\begin{aligned}
& P_{\delta}\left(z^{l-1}\left(I_{0+}^{\zeta, \zeta^{\prime}, \varrho, \varrho^{\prime}, \kappa} t^{\chi-1} J_{\omega, \vartheta}^{\varphi, m}(t z)\right)(x): s\right)=\frac{x^{A-\zeta-\zeta^{\prime}+\kappa-1}}{s^{l} 2^{\omega+2 \vartheta}} \\
& \times{ }_{5} \psi_{4+m}\left[\begin{array}{c}
(A, 2),\left(A+\kappa-\zeta-\zeta^{\prime}-\varrho, 2\right),\left(A+\varrho^{\prime}-\zeta^{\prime}, 2\right), \\
(l+\omega+2 \vartheta, 2),(1,1)
\end{array} \mid \begin{array}{c}
x^{2} \\
\left(A+\varrho^{\prime}, 2\right),\left(\begin{array}{c}
\left.A+\kappa-\zeta-\zeta^{\prime}, 2\right),\left(A+\kappa-\zeta^{\prime}-\varrho, 2\right), \\
(\omega+\vartheta+1, \varphi), \underbrace{(\vartheta+1,1)}_{m-\text { times }}
\end{array}\right.
\end{array}\right]
\end{aligned}
$$

$$
\begin{aligned}
& P_{\delta}\left(z^{l-1}\left[I_{0-}^{\zeta, \zeta^{\prime}, \varrho, \varrho^{\prime}, \kappa} t^{\chi-1} J_{\omega, \vartheta}^{\varphi, m}(z / t)\right](x): s\right)=\frac{x^{\chi-\omega-2 \vartheta-\zeta-\zeta^{\prime}+\kappa-1}}{s^{l} 2^{\omega+2 \vartheta}} \\
& \times{ }_{5} \psi_{4+m}\left[\begin{array}{c|c}
\left(A-\kappa+\zeta+\zeta^{\prime}, 2\right),\left(A+\zeta+\varrho^{\prime}-\kappa, 2\right), \\
(A-\varrho, 2),(l+\omega+2 \vartheta, 2),(1,1) \\
(A, 2)\left(A+\zeta+\zeta^{\prime}+\varrho^{\prime}-\kappa, 2\right),(A+\zeta-\varrho, 2), \\
(\omega+\vartheta+1, \varphi), \underbrace{(\vartheta+1,1)}_{m-\text { times }}
\end{array}\right]-\frac{1}{s^{2 l} 4 x^{2}}
\end{aligned}
$$

where $A=1-\chi+\omega+2 \vartheta$.

\section{Remark 3.2.}

(1) On taking $m=1$, Theorems 3.3 and 3.4 lead to the $P_{\delta}$-transform formulas for fractional integrals of generalized Bessel function.

(2) A limit case of the Theorems 3.3 and 3.4, when $\delta \rightarrow 1$ and $m=1$, yields the Laplace transform formulas for fractional integrals of generalized Bessel function.

(3) On taking $m=1, \varphi=1$, and $\vartheta=\frac{1}{2}$, Theorems 3.3 and 3.4 yield the $P_{\delta}$-transform formulas for fractional integrals of Struve function.

(4) A limit case of Theorem 3.3 and 3.4, when $\delta \rightarrow 1$ and $m=1, \varphi=1$, and $\vartheta=\frac{1}{2}$, yield the Laplace transform formulas for fractional integrals of Struve function.

(5) On taking $m=1, \varphi=1$, and $\vartheta=0$, Theorem 3.3 and 3.4 yield the corresponding results for fractional integrals of Bessel function.

(6) A limit case of Theorem 3.3 when $\delta \rightarrow 1$ and $m=1, \varphi=$ 1 , and $\vartheta=0$ yield the corresponding Laplace transform formulas for fractional integrals of Bessel function.

\subsection{Image Formulas for Whittaker Transform}

Theorem 3.5. Let $\zeta, \zeta^{\prime}, \varrho, \varrho^{\prime}, \kappa, \vartheta, \eta, \sigma \in \mathbb{C}, m \in \mathbb{N}, \varphi>0$, and $x>0$ be such that

$$
\begin{gathered}
\Re(\kappa)>0, \Re(\omega)>-1, \mathfrak{R}(\tau \pm \eta)>-1 / 2, \\
\Re(\chi+\omega)>\max \left\{0, \Re\left(\zeta+\zeta^{\prime}+\varrho-\kappa\right), \mathfrak{R}\left(\zeta^{\prime}-\varrho^{\prime}\right)\right\}
\end{gathered}
$$

then the following Whittaker transform formula holds:

$$
\begin{aligned}
& \int_{0}^{\infty} z^{\sigma-1} e^{-z / 2}\left[W_{\sigma, \eta} I_{0+}^{\zeta, \zeta^{\prime}, \varrho, \varrho^{\prime}, \kappa}\left(t^{\chi-1} J_{\omega, \vartheta}^{\varphi, m}(z t)\right)(x)\right] d z \\
& =\frac{x^{A-\zeta-\zeta^{\prime}+\kappa-1}}{2^{\omega+2 \vartheta}} \\
& { }_{6} \psi_{5+m}\left[\begin{array}{c}
(A, 2),\left(A+\kappa-\zeta-\zeta^{\prime}-\varrho, 2\right),\left(A+\varrho^{\prime}-\zeta^{\prime}, 2\right), \\
(E+\eta, 2),(E-\eta, 2),(1,1) \\
\left(A+\varrho^{\prime}, 2\right),\left(A+\kappa-\zeta-\zeta^{\prime}, 2\right),\left(A+\kappa-\zeta^{\prime}-\varrho, 2\right), \mid \\
(\omega+\vartheta+1, \varphi),(E-\sigma, 2), \underbrace{(\vartheta+1,1)}_{m-\text { times }}
\end{array} \mid\right.
\end{aligned}
$$

where $A=\chi+\omega+2 \vartheta$. 
where $A=\chi+\omega+2 \vartheta$ and $E=\tau+\omega+2 \vartheta+1 / 2$.

Proof: For simplicity, let $\varpi$ be the left-hand side of the formula (3.14). Applying (1.35) to Equation (3.14), we have

$$
\varpi=\int_{0}^{\infty} z^{\sigma-1} e^{-z / 2} W_{\sigma, \eta}\left[I_{0+}^{\zeta, \zeta^{\prime}, \varrho, \varrho^{\prime}, \kappa}\left(t^{\chi-1} J_{\omega, \vartheta}^{\varphi, m}(z t)\right)(x)\right] d z .
$$

Here, applying Equation (2.2) to the integral, we obtain the following expression:

$$
\begin{aligned}
\varpi & =\int_{0}^{\infty} z^{\sigma+\omega+2 \vartheta-1} e^{-z / 2} W_{\sigma, \eta} \\
& {\left[\frac{x^{A-\zeta-\zeta^{\prime}+\kappa-1}}{2^{\omega+2 \vartheta}} \sum_{k=0}^{\infty} \frac{\Gamma(A+2 k) \Gamma\left(A+\kappa-\zeta-\zeta^{\prime}-\varrho+2 k\right)}{\Gamma\left(A+\varrho^{\prime}+2 k\right) \Gamma\left(A+\kappa-\zeta-\zeta^{\prime}+2 k\right)}\right.} \\
& \times \frac{\Gamma\left(A+\varrho^{\prime}-\zeta^{\prime}+2 k\right) \Gamma(k+1)(-1)^{k}}{\Gamma\left(A+\kappa-\zeta^{\prime}-\varrho+2 k\right) \Gamma(\omega+\vartheta+1+\varphi k)(\Gamma(\vartheta+1+k))^{m}} \\
& \left.\times \frac{(z x)^{2 k}}{4^{k} k !}\right] d z
\end{aligned}
$$

where $A=\chi+\omega+2 \vartheta$. Interchanging the order of integration and summation, we have
Proof: We can establish the result given in Theorem 3.6 similar to the proof of Theorem 3.5.

\section{Remark 3.3.}

(1) For $m=1$, Theorems 3.5 and 3.6 lead to the corresponding results for fractional integral of generalized Bessel function defined in (1.3).

(2) If we take $m=1, \varphi=1$, and $\vartheta=\frac{1}{2}$, Theorems 3.5 and 3.6 yield the corresponding results for fractional integral of Struve function defined in (1.4).

(3) On taking $m=1, \varphi=1$, and $\vartheta=0$, Theorems 3.5 and 3.6 yield the corresponding results for fractional integral of Bessel function defined in (1.5).

\section{SPECIAL CASES AND CONCLUDING REMARKS}

In this section, we consider some special cases of our main results involved in Theorems 2.1-3.6 which can be obtained by setting $\zeta^{\prime}=0$. These interesting corollaries of our results involve the Saigo fractional integral operators $I_{0, x}^{\zeta, \varrho, \eta}$ and $I_{x, \infty}^{\zeta, \varrho, \eta}$ and can be

$$
\begin{aligned}
\varpi & =\frac{x^{A-\zeta-\zeta^{\prime}+\kappa-1}}{2^{\omega+2 \vartheta}} \sum_{k=0}^{\infty} \frac{\Gamma(E+\eta+2 k) \Gamma(E-\eta+2 k) \Gamma\left(A+\kappa-\zeta-\zeta^{\prime}-\varrho+2 k\right)}{\Gamma(E-\sigma+2 k) \Gamma\left(A+\kappa-\zeta^{\prime}-\varrho+2 k\right)} \\
& \times \frac{(-1)^{k} \Gamma(A+2 k) \Gamma\left(A+\varrho^{\prime}-\zeta^{\prime}+2 k\right) \Gamma(k+1)}{\Gamma\left(A+\varrho^{\prime}+2 k\right) \Gamma\left(A+\kappa-\zeta-\zeta^{\prime}+2 k\right) \Gamma(\omega+\vartheta+1+\varphi k)(\Gamma(\vartheta+1+k))^{m}} \frac{x^{2 k}}{4^{k} k !}
\end{aligned}
$$

where $A=\chi+\omega+2 \vartheta$ and $E=\tau+\omega+2 \vartheta+1 / 2$.

Interpreting the right-hand side of the above equation, in view of the definition (1.2), we arrive at the required result (3.14).

Theorem 3.6. Let $\zeta, \zeta^{\prime}, \varrho, \varrho^{\prime}, \kappa, \vartheta, \eta, \sigma \in \mathbb{C}, m \in \mathbb{N}, \varphi>0$, and $x>0$ be such that

$$
\begin{gathered}
\Re(\kappa)>0, \mathfrak{R}(\omega)>-1, \mathfrak{R}(\tau \pm n)>-1 / 2, \\
\Re(\chi-\omega)>1+\min \left\{\mathfrak{R}(-\varrho), \mathfrak{R}\left(\zeta+\zeta^{\prime}-\kappa\right), \mathfrak{R}\left(\zeta+\varrho^{\prime}-\kappa\right)\right\}
\end{gathered}
$$

then there holds the formula

$$
\begin{aligned}
& \int_{0}^{\infty} z^{\sigma-1} e^{-z / 2} W_{\sigma, \eta}\left[\left(I_{0-}^{\zeta, \zeta^{\prime}, \varrho, \varrho^{\prime}, \kappa} t^{\chi-1} J_{\omega, \vartheta}^{\varphi, m}(z t)\right)(x)\right] d z \\
& \left.=\frac{x^{\chi-\omega-2 \vartheta-\zeta-\zeta^{\prime}+\kappa-1}}{{ }_{6} \psi_{5+m}\left[\begin{array}{c}
\left(A-\kappa+\zeta+\zeta^{\prime}, 2\right),\left(A+\zeta+\varrho^{\prime}-\kappa, 2\right), \\
(A-\varrho, 2),(E+\eta, 2),(E-\eta, 2),(1,1) \\
(A, 2)\left(A+\zeta+\zeta^{\prime}+\varrho^{\prime}-\kappa, 2\right),(A+\zeta-\varrho, 2), \\
(\omega+\vartheta+1, \varphi),(E-\sigma, 2), \underbrace{(\vartheta+1)}_{m-\text { times }}
\end{array} \mid\right.} \mid \begin{array}{c}
4 \\
4 x^{2}
\end{array}\right]
\end{aligned}
$$

where $A=1-\chi+\omega+2 \vartheta$ and $E=\tau+\omega+2 \vartheta+1 / 2$. deduced from the Theorems $2.1-3.6$ by appropriately applying the relationships given in the definitions (1.16) and (1.17). If we set $\varrho=-\zeta$ in the Theorems 2.1-3.6, then from the relationships (1.20) and (1.21) we obtain the corresponding results for the Riemann-Liouville and the Weyl fractional integral operators, respectively. Again, if we put $\varrho=0$ in the Theorems 2.13.6, then from the relationships (1.24) and (1.25) we obtain the analogous results for Erdélyi-Kober type fractional integral operators.

In our present investigation, we establish the relationship between well-known fractional integral operators with novel integral transforms. The results obtained here are useful in deriving at various image formulas. The results presented here are very generic and can be specialized to give further potentially interesting and useful formulas involving fractional integral operators.

\section{AUTHOR CONTRIBUTIONS}

RPA devised the problem and supervised the manuscript by adding various results to it. RA and SJ worked on the mathematics in the manuscript. DB provided guidance, checked all calculations, and suggested language modifications to the article paper. 


\section{REFERENCES}

1. Atangana A, Jain S. A new numerical approximation of the fractal ordinary differential equation. Eur Phys J Plus (2018) 133:37. doi: 10.1140/epjp/i201811895-1

2. Jain S. Numerical analysis for the fractional diffusion and fractional Buckmaster's equation by two step Adam- Bashforth method. Eur Phys J Plus (2018) 133:19 doi: 10.1140/epjp/i2018-11854-x

3. Agarwal R, Jain S, Agarwal RP. Analytic solution of generalized space time advection-dispersion equation with fractional Laplace operator. J Nonlinear Sci Appl. (2016) 9:3545-54. doi: 10.22436/jnsa.009.06.09

4. Agarwal R, Jain S, Agarwal RP. Analytic solution of generalized space time fractional fraction diffusion equation. Fract Differ Calc. (2017) 7:169-84. doi: 10.7153/fdc-07-05

5. Samko SG, Kilbas AA, Marichev OI. Fractional Integrals and Derivatives: Theory and Applications New York, NY: Gordon \& Breach Science Publishers Inc. (1993).

6. Saxena RK, Saigo M. Generalized fractional calculus of the H-function associated with the Appell function. J Frac Calc. (2001) 19:89-104.

7. Srivastava HM, Tomovski Z. Fractional calculus with an integral operator containing a generalized Mittag-Leffler function in the kernel. Appl Math Comput. (2009) 211:198-210. doi: 10.1016/j.amc.2009.01.055

8. Srivastava HM, Saxena RK. Operators of fractional integration and their applications. Appl Math Comput. (2001) 118:1-52 doi: 10.1016/S0096-3003(99)00208-8

9. Oteiza MBM, de Kalla S, Conde S. Un estudio sobre la funcition LommelMaitland. Rev Técnica Facult Ingenieria Univers Zulia (1986) 9:33-40.

10. Srivastava HM, Karlsson PW. Multiple Gaussian Hypergeometric Series. New York, NY; Chichester; Brisbane, QLD; Toronto, ON: Halsted Press; Ellis Horwood Limited; John Wiley and Sons (1985).

11. Kilbas AA, Srivastava HM, Trujillo JJ. Theory and Applications of Fractional Differential Equations. Amsterdem: Elsevier Science; NorthHolland Mathematical Studies (2006).

12. Prieto AI, de Romero SS, Srivastava HM. Some fractional calculus results involving the generalized Lommel-Wright and related functions. Appl Math Lett. (2007) 20:17-22. doi: 10.1016/j.aml.2006.02.018

13. Paneva-Konovska J. Theorems on the convergence of series in genearlized Lommel-Wright functions. Frac Cal Appl Anal. (2007) 10:60-74.

14. Pathak RS. Certain convergence theorems and asymptotic properties of a generalization of Lommel and Maitland transformations. Proc Nat Acad Sci USA. (1966) A-36:81-6.

15. Kiryakova V. On two Saigo's fractional integral operators in the class of univalent functions. Fract Calc Appl Anal. (2006) 9:159-76.

16. Mathai AM, Saxena RK, Haubold HJ. The H-Function Theory and Applications. New York, NY: Springer-Verlag (2010).
17. Saigo M. A remark on integral operators involving the Gauss hypergeometric functions. Math Rep Kyushu Univ. (1978) 11:135-43.

18. Saigo M. A certain boundary value problem for the Euler-Darboux equation I. Math Japonica (1979) 24:377-85.

19. Marichev OI. Volterra equation of Mellin convolution type with a Horn function in the kernel (In Russian). Izv AN BSSR Ser Fiz-Mat Nauk (1974) 1:128-9.

20. Saigo M, Maeda N. More generalization of fractional calculus. In: Transform Methods and Special Functions. Varna: Bulgarian Academy of Science (1998).

21. Oldham KB, Spanier J. The Fractional Calculus: Theory and Applications of Differentiation and Integration of Arbitrary Order. New York, NY: Academic Press (1974).

22. Kober H. On fractional integrals and derivatives. Quart J Math Oxford Ser. (1940) 11:193-212.

23. Sneddon IN. The Use of Integral Transforms. New Delhi: Tata McGraw-Hill (1979).

24. Kumar D. Solution of fractional kinetic equation by a class of integral transform of pathway type. J Math Phys. (2013) 54:043509. doi: $10.1063 / 1.4800768$

25. Agarwal R, Jain S, Agarwal RP. Solution of fractional Volterra integral equation and non-homogeneous time fractional heat equation using integral transform of pathway type. Progr Fract Differ Appl. (2015) 1:145-55. doi: $10.12785 / \mathrm{pfda} / 010301$

26. Srivastava HM, Agarwal R, Jain S. Integral transform and fractional derivative formulas involving the extended generalized hypergeometric functions and probability distributions Math Methods Appl Sci. (2017) 40:255-73. doi: 10.1002/mma.3986

27. Srivastava R, Agarwal R, Jain S. A family of the incomplete hypergeometric functions and associated integral transform and fractional derivative formulas. Filomat (2017) 31:125-40. doi: 10.2298/FIL170 $1125 \mathrm{~S}$

28. Purohit SD, Suthar DL, Kalla SL. Marichev-Saigo-Maeda fractional integration operators of the Bessel functions. Matematiche (2012) 67:21-32.

Conflict of Interest Statement: The authors declare that the research was conducted in the absence of any commercial or financial relationships that could be construed as a potential conflict of interest.

Copyright (C) 2018 Agarwal, Jain, Agarwal and Baleanu. This is an open-access article distributed under the terms of the Creative Commons Attribution License (CC BY). The use, distribution or reproduction in other forums is permitted, provided the original author(s) and the copyright owner(s) are credited and that the original publication in this journal is cited, in accordance with accepted academic practice. No use, distribution or reproduction is permitted which does not comply with these terms. 